\title{
Harmonics of whistler-mode waves near the Moon
}

\author{
Yasunori Tsugawa ${ }^{1 *}$, Yuto Katoh', Naoki Terada ${ }^{1}$, Hideo Tsunakawa², Futoshi Takahashi ${ }^{3}$, Hidetoshi Shibuya ${ }^{4}$, \\ Hisayoshi Shimizu ${ }^{5}$ and Masaki Matsushima ${ }^{2}$
}

\begin{abstract}
Harmonic spectral features of electromagnetic waves in the frequencies of several $\mathrm{Hz}$ around the Moon have been identified by Kaguya. The waves have steepened waveforms peculiarly in the compressional component. The fundamental waves have almost the same properties as narrowband whistler-mode waves with the frequencies near $1 \mathrm{~Hz}$, which have been observed around the Moon. The waves are observed around the terminator region in the solar wind near the lunar magnetic anomalies at the altitudes under $120 \mathrm{~km}$. We suggest that the harmonic spectra are a result of the nonlinear steepening of narrowband whistler-mode waves. Although the narrowband whistler-mode waves have been observed in the upstream region of many planetary bow shocks, such harmonics have rarely been observed there. Since the harmonics are more frequently observed at lower altitudes of the Moon, they are possibly caused by lunar intrinsic environments including lunar dusts and local structures of lunar magnetic anomalies.
\end{abstract}

Keywords: Harmonic wave; Nonlinear steepening; Whistler-mode; Solar wind; Magnetic anomaly; Lunar dust

\section{Findings}

Introduction

The Moon is characterized as a less conductive body with a tenuous atmosphere, small-scale crustal magnetic anomalies, and no global magnetic field. The lunar environment is controlled by the interactions with ambient plasmas, which cause some intrinsic phenomena including mini-magnetospheres around the magnetic anomalies and levitation of lunar dusts. These should significantly affect the lunar ambient environment in the plasma state and electromagnetic field.

Upstream whistler-mode waves with narrowband spectra in the frequencies near $1 \mathrm{~Hz}$ have been observed in the solar wind around the Moon (Halekas et al. 2006; Tsugawa et al. 2011) and many solar system bodies (Orlowski and Russell 1995; Russell 2007). They are mostly left-hand polarized in the spacecraft frame due to large Doppler shift by the solar wind (Fairfield 1974). The similarities of the wave properties throughout the solar system imply that the wave formation processes are independent of the shape

\footnotetext{
* Correspondence: yasunori@stpp.gp.tohoku.ac.jp

${ }^{1}$ Department of Geophysics, Graduate School of Science, Tohoku University, 6-3 Aramaki-aza-aoba, Aoba, Sendai, Miyagi 980-8578, Japan Full list of author information is available at the end of the article
}

and size of the obstacle (Orlowski and Russell 1995). Recently, Tsugawa et al. (2014) proposed that the waves are group-standing, i.e., they are nearly stagnated in the spacecraft frame. The wave properties observed near the Moon can be explained by the group-standing condition, indicating that the narrowband spectra are apparent in the spacecraft frame through the spectral modification by the group-standing effect (Tsugawa et al. 2014). However, the generation processes and other effects on the waves have not been fully understood.

In this paper, we present harmonics of the upstream whistler-mode waves for the first time observed near the Moon by Kaguya. The harmonics are possibly explained by the nonlinear phase steepening of waves that appeared at the fundamental frequency with a finite compressional component. Even in a plasma medium, the phase steepening is common development of compressive waves with large amplitude in mostly linear dispersive wave mode, such as ion acoustic mode and magnetosonic mode. However, the observation of steepened waves in the frequency range of whistler mode has rarely been reported, possibly because of the dispersion and dissipation effects on the harmonic components (e.g., Lembege 2003). The former effect works on the harmonics to radiate from the fundamental

\section{它}


waves. The latter effect includes a variety of interactions with particles, which attenuate the harmonics. These effects sensitively depend on the wave properties and the ambient plasma state, such as the wave frequency, wavelength, amplitude, propagation angle, components of plasma particles, and their velocity distributions. Kellogg et al. (2011) reported that recently discovered obliquely propagating large-amplitude whistler-mode waves in the inner terrestrial magnetosphere (Cattell et al. 2008) have been observed with their second harmonics in the electrostatic components and are associated with the electron trapping by the electrostatic fields. While Yoon et al. (2014) pointed out the significance of the initial wave amplitude and wave propagation angle for the phase steepening of large-amplitude whistler-mode waves, the condition to steepen and the influences of ambient plasma have rarely been clarified.

Even though the narrowband upstream whistler-mode waves have been observed for four decades around various bodies in the solar wind (Orlowski and Russell 1995), their harmonics have never been reported. It seems to be natural that the waves hardly steepen since their narrowband spectra in the spacecraft frame can be formed from waves in the plasma frame with a broader frequency band and smaller amplitudes than those of observed waves. Our finding of the harmonics of the waves near the Moon suggests the existence of additional effects on the waves. In the present study, we investigate characteristic properties of the harmonic waves near the Moon so as to provide clues for understanding the physics of the nonlinear development of whistler-mode waves and the causes associated with the lunar intrinsic environments.

\section{Observations}

The SELENE (Kaguya) spacecraft orbited around the Moon at altitudes of about $100 \mathrm{~km}$ in the range from 80 to $120 \mathrm{~km}$ from October 2007 to December 2008 and then at lower altitudes to June 2009 in a polar orbit. The lunar magnetometer (LMAG) on Kaguya measured the magnetic field vectors at a sampling rate of $32 \mathrm{~Hz}$ (Tsunakawa et al. 2010). We performed fast Fourier transformation (FFT) to the magnetic field vectors in every $16 \mathrm{~s}$ to analyze wave activities in the frequencies from 0.0625 to $16 \mathrm{~Hz}$.

Figure 1 shows an example of the harmonic waves detected by Kaguya on 13 June 2008. Narrowband waves were observed in the frequencies near $1 \mathrm{~Hz}$ during 00:05 to 00:40 UT (Figure 1a). The second and third harmonic spectra of the narrowband waves were observed during 00:10 to $00: 40$ and during 00:10 to 00:30, respectively. The frequency intervals of the harmonics are almost equivalent (approximately $1.2 \mathrm{~Hz}$ ) as shown in Figure $1 \mathrm{~g}$. The compressional components $(\mathrm{C})$ of the second and third harmonics are one order of magnitude larger than the rotational components $(\mathrm{R}, \mathrm{L})$. The intensities of the harmonic components decrease with the increase of the frequency. Figure $1 \mathrm{~h}$ shows the waveform of the two transverse and the compressional components with respect to the mean field during 00:28:40 to 00:29:00. All of the components, particularly the compressional component, show steepened waveforms. The intensity of the compressional component is inversely proportional to the 4th power of the harmonic number (Figure 1g), consistently with the steepened waveform. The ratio of the wave amplitudes of the fundamental waves with respect to the ambient magnetic field strength is approximately 0.2 to 0.3 .

Figure $1 \mathrm{~b}$ shows that the fundamental waves are mostly left-hand polarized. During most of this period, the sunward $(x)$ component of the magnetic field is larger than the other components as shown in Figure 1e, indicating that the magnetic field line lay nearly parallel to the solar wind flow. Kaguya orbited near the terminator region at an altitude near $100 \mathrm{~km}$ in this period as shown in Figure 1f. These features of the fundamental waves and conditions are the same as the narrowband whistler-mode waves (Tsugawa et al. 2014). Figure 1c, d indicates the angle between the wave vector and sunward directions $\left(\theta_{k x}\right)$ and the angle between the wave vector and magnetic field directions $\left(\theta_{k x}\right)$, respectively. The wave vector directions are estimated by minimum variance analysis (MVA) (Sonnerup and Scheible 1998). In contrast to most of the narrowband waves, the wave vectors of the fundamental waves direct slightly large angles with respect to the sunward and magnetic field directions $\left(\theta_{k x} \sim 40^{\circ}\right.$ to $60^{\circ}, \theta_{k B} \sim 50^{\circ}$ to $\left.70^{\circ}\right)$ as shown in Figure $1 \mathrm{c}, \mathrm{d}$.

\section{Statistical properties}

We selected the harmonic waves by analyzing dataset from 1 December 2007 to 10 June 2009 in order to investigate their statistical properties. In that period, Kaguya orbited at altitudes under $120 \mathrm{~km}$ : around $100 \mathrm{~km}$ in 2008 and at lower altitudes in 2009. We first identified the fundamental narrowband waves using basically the same criteria we used in previous studies (Tsugawa et al. 2011, 2014) and then investigated the presence of harmonics whose amplitudes are larger than the noise level referring to clear harmonic spectra as shown in Figure $1 \mathrm{~g}$ by applying the following criteria:

1) The fundamental wave has a peak intensity between 0.4 and $4 \mathrm{~Hz}$ whose frequency is defined as $f_{\text {peak }}(\mathrm{nh}=1)$, where nh represents the number of harmonics.

2) The harmonic frequencies $f_{\text {peak }}(\mathrm{nh} \geq 2)$ are determined as the frequencies in which the intensity has a peak in the frequency range from $(\mathrm{nh}-2 / 5)$ $f_{\text {peak }}(1)$ to $(\mathrm{nh}+2 / 5) f_{\text {peak }}(1)$. 

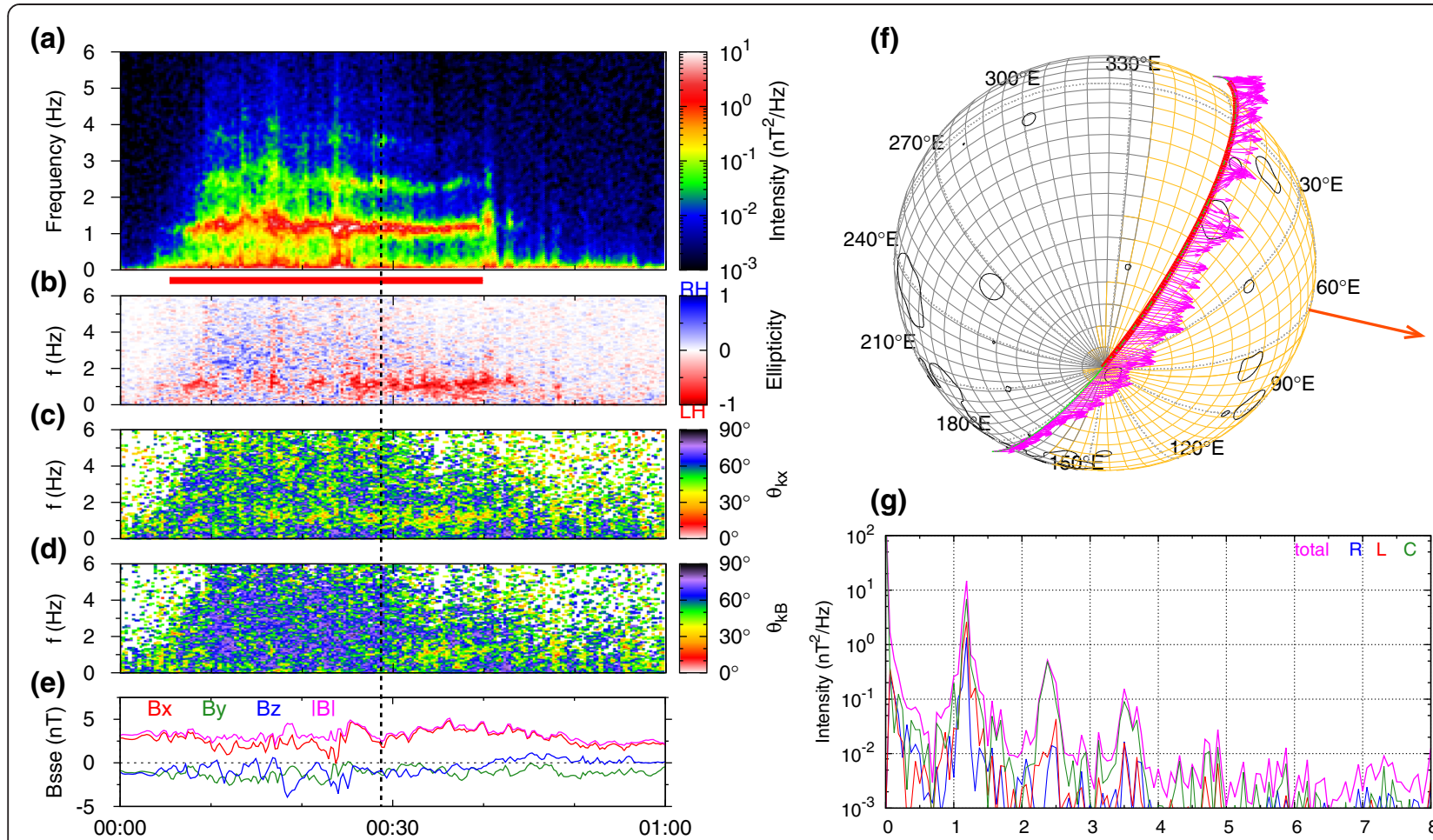

(g)

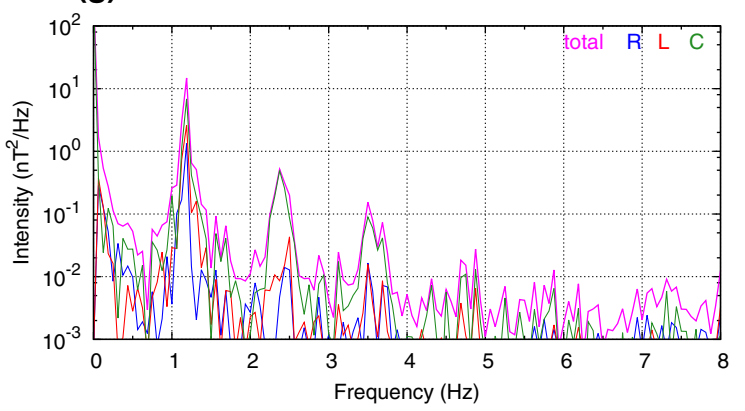

(h)

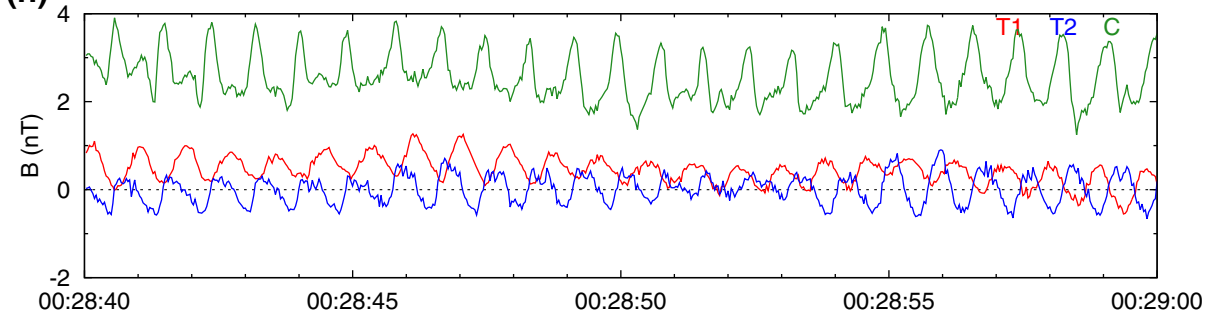

Figure 1 The harmonic waves detected by Kaguya on 13 June 2008. (a) The fast Fourier transform (FFT) spectrum of magnetic field. (b) The ellipticity spectrum. The angles (c) between the wave vector and the sunward direction $\left(\theta_{k x}\right)$ and (d) between the wave vector and the magnetic field $\left(\theta_{k B}\right)$. (e) The magnetic field vector components in the Selenocentric Solar Ecliptic (SSE) coordinates. Ellipticity is defined as $(R-L) /(R+L)$, where $R$ and $L$ represent the right- and left-hand polarized components of the intensity of the waves, respectively. (f) The spacecraft trajectory during 00:00 to 01:00 UT (green line) and magnetic field vectors (magenta arrows) with respect to the Moon. The black contour indicates 0.5-nT magnetic field strength at an altitude of $100 \mathrm{~km}$ (Tsunakawa et al. 2010). The yellow and gray hemispheres in (f) represent the dayside and nightside of the Moon, respectively. The orange arrow represents the sunward direction from the subsolar point. The thick red line superposed on the trajectory represents the period during 00:05 to 00:40 UT indicated by a red bar just below (a). (g) The total, R, L, and compressional (C) components of the wave intensity versus the frequency at 00:29 UT. (h) The waveform of the two transverse $(\mathrm{T} 1, \mathrm{~T} 2)$ and $\mathrm{C}$ components with respect to the mean field during 00:28:40 to 00:29:00 UT.

3) The intensities of harmonics are larger than the noise level, $0.016 / \mathrm{nh}^{2}$.

4) The intensities of $f_{\text {peak }}(\mathrm{nh})$ have drop levels larger than $\left(4 / \mathrm{nh}^{2}+4\right) \mathrm{nT}^{2} / \mathrm{Hz}$ in the frequency range from $f_{\text {peak }}(\mathrm{nh}-1)$ to $f_{\text {peak }}(\mathrm{nh})$.

The harmonic waves were so rarely observed that the number of clear events is about 30 in the whole analyzed time. We have confirmed that these criteria can predominantly select the clear harmonic waves. Applying these criteria, we selected 1,884 interval points of FFT, which correspond to the total duration of $8.4 \mathrm{~h}$ and $0.10 \%$ of the analyzed period.

\section{Fundamental and harmonic components}

As mentioned above, the fundamental waves resemble to narrowband whistler-mode waves. Firstly, we examine the observed frequency and polarization of the fundamental waves with respect to the angle between the wave vector and the sunward direction $\left(\theta_{k x}\right)$, which corresponds to the significance of the Doppler shift on the wave spectra (Figure 2). The wave vector directions of 


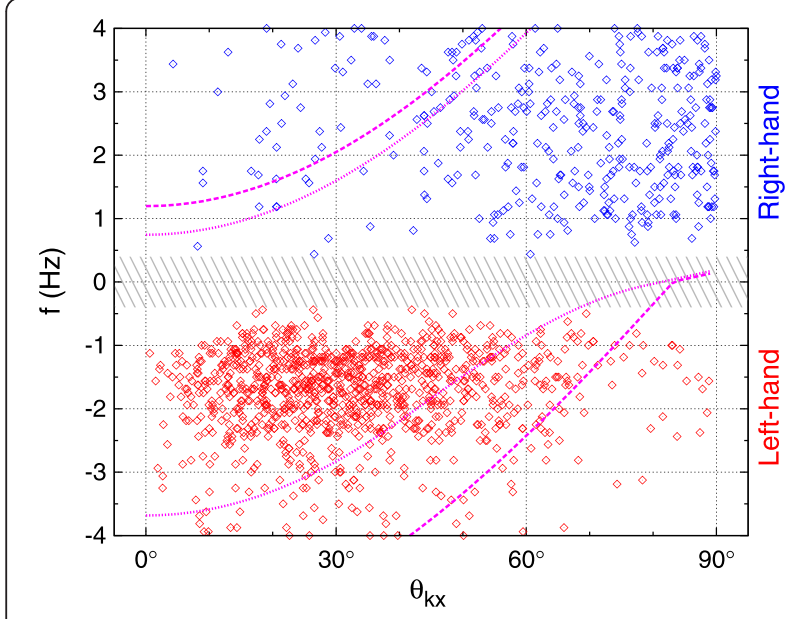

Figure 2 Frequencies of the fundamental waves as a function of $\boldsymbol{\theta}_{\boldsymbol{k} \boldsymbol{x}}$. The frequencies of right- and left-hand polarized waves are defined as positive and negative values, respectively. The magenta dashed lines represent the Doppler-shifted frequency range calculated for the whistler-mode waves with $k<0.1 / \mathrm{km}, \theta_{k B}=0^{\circ}$ to $90^{\circ}$ in $B=5 \mathrm{nT}$, $n=5 / \mathrm{cm}^{3}, V_{s w}=400 \mathrm{~km} / \mathrm{s}$. The magenta dotted lines represent the same as the dashed lines but with $\theta_{k B}=20^{\circ}$ to $70^{\circ}$.

the fundamental waves are obtained by MVA on inverse FFT waveform of the frequency components of the fundamental waves. Figure 2 shows that the fundamental waves have a distribution closely similar to that of the narrowband waves, which are left-hand polarized at small $\theta_{k x}$ as shown in Figure four of Halekas et al. (2006). The distribution indicates that they are whistlermode waves propagating upstream with the wavelength on the order of $100 \mathrm{~km}$ and with the frequency around the lower hybrid resonance frequency in a typical solar wind condition at $1 \mathrm{AU}$.

Figure 3a shows the dependences of the occurrence of the harmonic waves on the ambient magnetic field direction, which are almost the same as those of the narrowband waves as shown in Figure two (c) of Tsugawa et al. (2014). Both these waves are mostly observed when the magnetic field directs about $20^{\circ}$ with respect to the solar wind flow direction. Next, we investigate dependences on the wave vector directions of the fundamental waves. The wave vector directions of the fundamental waves with respect to the magnetic field direction $\left(\theta_{k B}\right)$ (Figure $3 \mathrm{~b}$ ) are also analogous to those of the narrowband waves as shown in Figure two (b) of Tsugawa et al. (2014). Moreover, Figure 3b indicates that $\theta_{k B}$ have a positive correlation with $\theta_{k x}$ in a similar manner to the narrowband waves (Figure three of Tsugawa et al. (2014)). These results suggest that the fundamental waves are basically identical to the narrowband whistlermode waves, which are considered as group-standing whistler-mode waves, nearly stagnating in the spacecraft frame (Tsugawa et al. 2014). This implies that a fraction of narrowband waves are observed with their harmonics in a specific condition.

Figure 4 shows the properties of the harmonic components. As with the waves shown in Figure 1, the harmonics mostly have the frequencies of integer multiples of the fundamental waves. Since we selected the harmonic waves by a modest fitting to obtain a certain amount of events for the statistical study, the frequencies of the harmonics disperse around the integer multiples of the fundamental waves. The intensities of the harmonics are reduced roughly in a manner proportional to the negative 4th power of the harmonic number, which can exhibit the steepened waveform. The harmonics almost consist of compressional components as shown in Figure 4.

\section{Spatial distribution}

Figure 5 shows the spatial distributions of the harmonic waves observed by Kaguya at altitudes under $120 \mathrm{~km}$. Figure $5 \mathrm{a}, \mathrm{b}$ shows the locations where the harmonic waves were observed in each analyzed interval, $16 \mathrm{~s}$. Figure $5 \mathrm{a}$ indicates that the harmonic waves were mostly observed in the dayside. The waves with a higher intensity and larger harmonic number were observed closer to the terminator region (Figure 5a) and closer to the magnetic

(a)

(b)

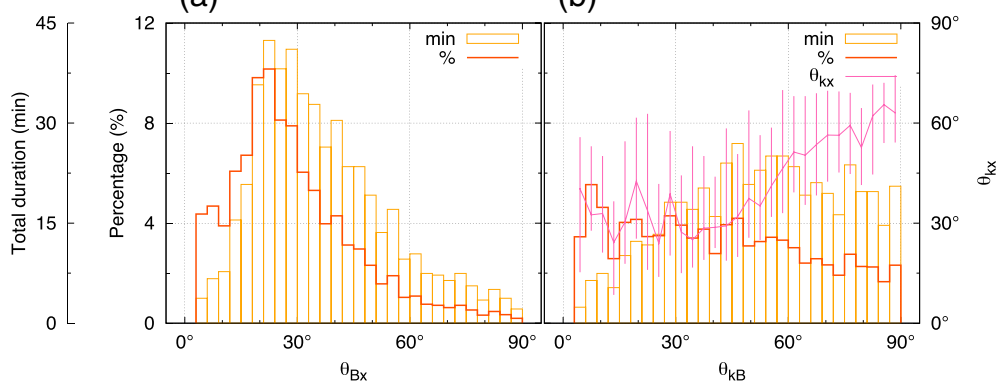

Figure 3 Dependences of the harmonic waves on the magnetic field, sunward, and wave vector directions. The horizontal axes are (a) $\theta_{B x}$ and (b) $\theta_{k B}$. The total durations are in minutes. The percentage is derived from total durations normalized by the solid angle of each bin. 


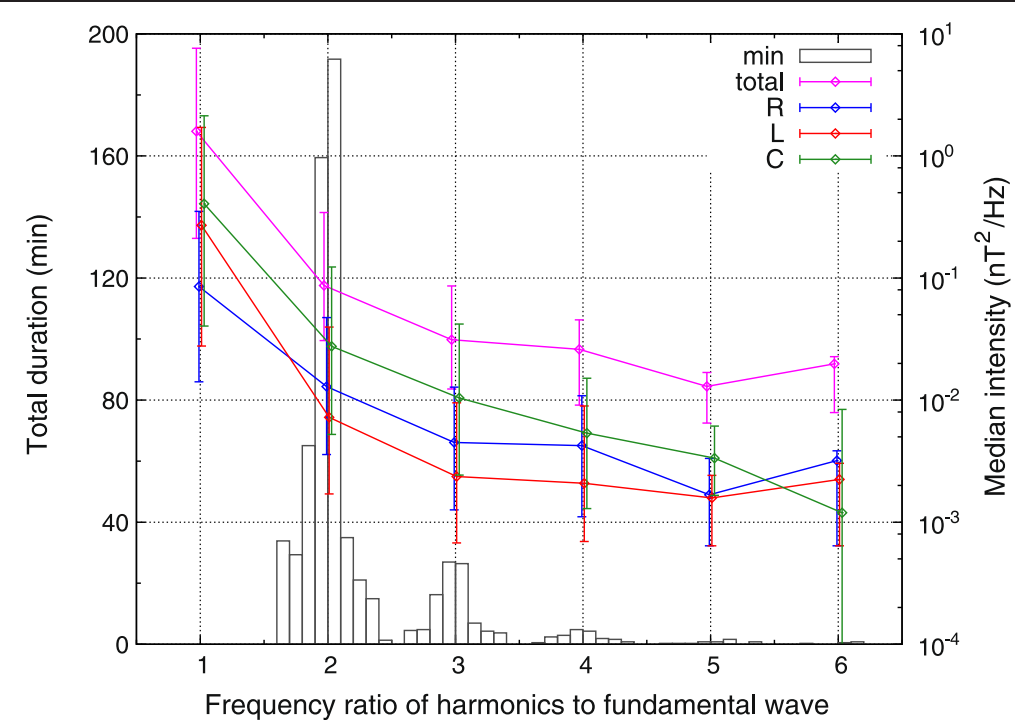

Figure 4 Dependences of the harmonic waves on the frequency ratio of harmonics to the fundamental waves. The intensities are median values of the total, right-hand (R)/left-hand (L) polarized, and compressional (C) components. The errors of the intensities represent quartiles around the median.

anomalies (Figure 5b). This tendency may reflect the nature of the waves, though the observed locations distribute over a wide area.

Figure $5 \mathrm{c}, \mathrm{d}$ represents the occurrence rate distributions of the harmonic waves in the analyzed period, which are derived from dividing the total durations of the waves with harmonics (as shown in Figure 5a,b, respectively) by the total analyzed durations in each bin. The results indicate that the waves were mostly observed at the solar zenith angle (SZA) of $40^{\circ}$ to $90^{\circ}$ (Figure 5c) and near the magnetic anomalies (Figure $5 \mathrm{~d}$ ), where the narrowband waves are also frequently observed (Figure two of Tsugawa et al. (2011)). In particular, the higher occurrence regions above $0.7 \%$ are localized in some spots of the largest anomalies around the South Pole Aitken basin region, which is centered at $55^{\circ} \mathrm{S}, 180^{\circ} \mathrm{E}$, as shown in Figure 5d.

To compare the distributions of the harmonic waves and the narrowband waves, we show their superposition in Figure 5e,f. The occurrence distribution of the narrowband waves are the same as that in Figure two of Tsugawa et al. (2011), but the end of the analyzed period is extended from September 2008 to June 2009. Though the distributions of the harmonic waves and the narrowband waves are basically similar in both SSE and ME coordinates, the higher occurrence regions of the harmonic waves are more localized than those of the narrowband waves. In ME coordinates, the harmonic waves are frequently observed at regions closer to the strong magnetic anomalies than the narrowband waves as shown in Figure $5 f$.

\section{Discussion}

\section{Possible causes of harmonics}

Before discussing physical interpretations of the waves, we assess instrumental and analytical influences on the wave spectra. Since the dynamic range of LMAG during the analyzed period was fixed in a range of $\pm 64 \mathrm{nT}$ (Tsunakawa et al. 2010), effects of different offsets and linearities between ranges did not affect the output. The linearity of the sensor in a range of $\pm 64 \mathrm{nT}$ was confirmed to be better than $0.2 \%$ by the ground calibration (Shimizu et al. 2008). Therefore, the distortion of output waveforms was negligible compared with the observed harmonics. The measurement system of LMAG could generate an instrumental noise (Takahashi et al. 2009), which is estimated to be about $10^{-4}$ of the external field and thus negligible. LMAG also detected weak instrumental noises in several integer frequencies. Since the noises have at most $10^{-2} \mathrm{nT}^{2} / \mathrm{Hz}$ and their frequencies are constant in time, they can be ignored compared with the observed harmonics. The discontinuity of boundaries of the FFT analysis can also show apparent harmonics. The periods of the relevant fundamental waves are at most approximately $1 \mathrm{~s}$, which are enough shorter than the analyzed period, $16 \mathrm{~s}$. In such case, the apparent harmonics of the relevant waves formed by FFT analysis are under the noise level. We thereby reject instrumental and analytical issues for the generation of the harmonic waves.

For a physical process associated with the harmonic waves, one would mind associations with the cyclotron harmonics of charged particles. Electromagnetic ion cyclotron waves can be observed as left-hand polarized 


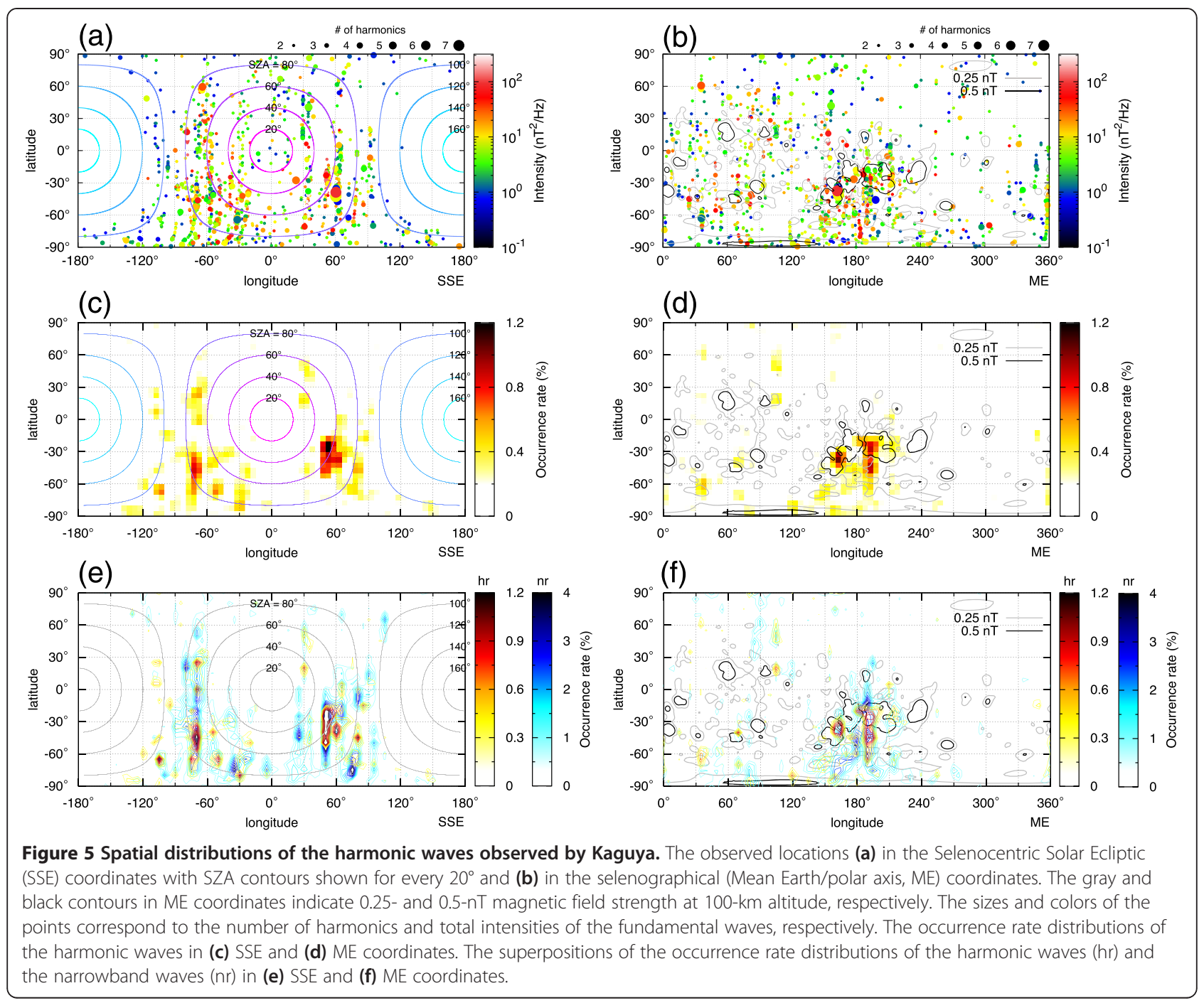

waves with harmonics in the observed frequency range when the wave vector directs anti-sunward and the frequency is positively Doppler shifted by the solar wind flow. However, the cyclotron harmonics would not be related to the observed waves, since the fundamental waves should lie in whistler mode as suggested in the 'Fundamental and harmonic components' section. Wavewave coupling might be able to form the harmonic spectra. In such process, the third wave $\left(\omega_{3}, \mathbf{k}_{3}\right)$ is generated satisfying a manner of $\omega_{3}=\omega_{1}+\omega_{2}$ in the frequencies and $\mathbf{k}_{3}=\mathbf{k}_{1}+\mathbf{k}_{2}$ in the wave vector, where $\left(\omega_{1}, \mathbf{k}_{1}\right)$ and $\left(\omega_{2}, \mathbf{k}_{2}\right)$ represent the parent waves. If both parent waves are the narrowband whistler-mode waves, the generated wave would have twice the frequency of the narrowband whistler-mode waves, and then the coupling between the parent and generated waves might produce multiple frequency waves. However, since the process needs the dispersion relation satisfying the coupling manner and the wave amplitude larger than a threshold level, it is difficult to explain the observed properties of the harmonic spectra by the wave-wave coupling.

Nonlinear steepening can also form harmonic spectra. When a wave with finite amplitude modifies the medium state and the phase velocity depending on the wave phase, the wave components with a higher phase velocity propagate faster than the wave components with a lower phase velocity and the waveform is steepened. The steepened waves consist of harmonic components $(n \omega, n \mathbf{k})$ where $n$ is the harmonic number. The compressional component of the waves modifies the phase velocity and determines the significance of the nonlinear steepening. The harmonics related to steepened waves should have intensities decreasing with the harmonic number in a uniform manner. Actually, the waveform of the observed harmonic waves is clearly steepened in the compressional component and the intensities of the harmonics are decreased in a manner proportional to the negative 4 th power of the harmonic 
number as shown in Figure 1h. Since these features are also supported by statistical analysis as shown in Figure 4, we consider that the waves are nonlinearly steepened.

If the harmonics are caused by nonlinear steepening, their occurrence should depend on the intensity of the compressional component. Figure 6 shows the total durations of the harmonic waves with respect to the intensity ratio of the compressional component to the ambient magnetic field. Although the waves with a larger intensity ratio of the compressional components do not always exhibit the higher harmonics, the higher harmonics are typically more observed when the intensity ratio of the compressional components is larger. The waves with harmonics have a distribution of the intensity ratio of the compressional components with the median of $0.021 \mathrm{nT}^{2} / \mathrm{nT}^{2} \mathrm{~Hz}$ and quartiles of 0.029 and $0.095 \mathrm{nT}^{2} / \mathrm{nT}^{2} \mathrm{~Hz}$. The waves without harmonics have a distribution of the compressional components with the median of $0.0083 \mathrm{nT}^{2} / \mathrm{nT}^{2} \mathrm{~Hz}$ and quartiles of 0.0020 and $0.030 \mathrm{nT}^{2} / \mathrm{nT}^{2} \mathrm{~Hz}$. These suggest that the harmonic waves tend to be observed with a larger compressional component with respect to the ambient magnetic field strength, which is basically consistent with the nonlinear steepening. However, the waves with a larger intensity ratio of the compressional component did not always exhibit the harmonics as shown in Figure 6, suggesting that the condition to steepen is not significantly controlled only by the intensity ratio of the compressional components. It is notable that the development of the nonlinearity would also need both the propagation space and time in which the harmonics are not damped but the waveform can steepen considerably. Moreover, the modest fitting used in the

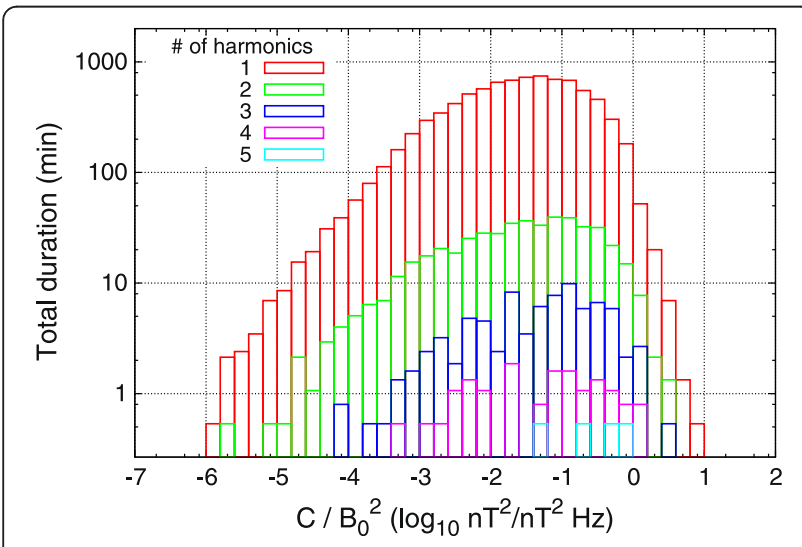

Figure 6 Dependences of the harmonic waves on the compressional intensity ratio to the background. The intensity ratio is obtained by dividing the compressional component of the wave intensity $(C)$ by the square of the ambient magnetic field strength averaged over the corresponding FFT period $\left(\mathrm{B}_{0}^{2}\right)$. Each color box represents the duration of the corresponding number of harmonics. The red boxes (\# of harmonics = 1) indicate the narrowband fundamental waves without higher harmonics. present study to select the harmonic waves possibly includes some irrelevant waves and might smooth the characteristic properties.

\section{Effects of lunar intrinsic environment}

The occurrence rate of the harmonic waves differs in the observed altitudes. They are more observed at lower altitudes. The narrowband waves are observed for $614 \mathrm{~min}$ and $29 \%$ of them have their harmonics at altitudes below $80 \mathrm{~km}$, whereas they are observed for $1,826 \mathrm{~min}$ and $18 \%$ of them have their harmonics above $80 \mathrm{~km}$. The high-occurrence regions of the harmonic waves below $80 \mathrm{~km}$ are more localized in some spots of the magnetic anomalies and distribute more widely in the dayside even at a smaller SZA region than those above $80 \mathrm{~km}$. These results suggest that some effects which work well closer to the Moon might contribute wave steepening.

Additionally, we revealed that such harmonics in the frequency of several $\mathrm{Hz}$ are rarely observed in the upstream region of the terrestrial bow shock, whereas the narrowband waves referred as ' $1 \mathrm{~Hz}$ waves' are frequently observed there. We investigated the dataset of Geotail which covers the entire region of the upstream region of the terrestrial bow shock. Geotail repeatedly crossed the wave source regions near the bow shock region about 400 times in 1995 to 1998. Analyzing the dataset of that period, we identified the narrowband waves of the total duration comparable to those observed by Kaguya (Tsugawa et al., in preparation). The properties of the narrowband waves observed by Geotail at the foreshock are almost similar to those observed by Kaguya near the Moon. The $\theta_{k B}$ and also the compressional components of the waves at the foreshock are slightly smaller than those of the waves near the Moon, suggesting the difficulty for the nonlinear steepening at the foreshock. However, it is peculiar that the harmonics have never been observed by Geotail, though some of the compressional components of the narrowband waves at the foreshock region are larger than those of the harmonic waves near the Moon. These features suggest that the generation process of the harmonic waves is strongly influenced by the intrinsic environment of the Moon.

The Moon has a very dusty environment and the charged dusts are lofted by the surface potentials. They would reach up to altitudes about $100 \mathrm{~km}$ near the terminator region, relatively higher than the dayside (e.g., Stubbs et al. 2006). Such charged dusts can modify the plasma medium to lead the wave steepening by reducing Landau damping (e.g., Luo et al. 1999). The high occurrence regions of the harmonic waves roughly agree with the dusty region, which are near the terminator at the higher altitudes approximately $100 \mathrm{~km}$ and even in the dayside at lower altitudes. Thus, the dusts possibly affect the wave steepening. The local plasma inhomogeneities around the 
magnetic anomalies would also contribute to the harmonic waves. The inhomogeneities can refract the waves (Katoh 2014) and make them compressive to develop the nonlinearity (Hada et al. 1987). Furthermore, cusp-like configurations of the magnetic anomalies might enhance the wave energy resulting in the anomalous development of the nonlinearity.

\section{Summary}

We revealed the existence of harmonic waves in the frequencies of several $\mathrm{Hz}$ near the Moon. The waveforms are steepened particularly in the compressional components. The waves are observed when the spacecraft orbits near the magnetic anomalies at SZA from $40^{\circ}$ to $90^{\circ}$ at altitudes under $120 \mathrm{~km}$. The fundamental waves are almost identical to the narrowband whistler-mode waves, which have been commonly observed around the Moon and in the upstream regions of solar system bodies. Since such harmonic waves are observed only near the Moon and the occurrence is higher at lower altitudes, they are possibly caused from lunar intrinsic environment. Lunar dusts and local structures of magnetic anomalies might promote the nonlinear steepening of the waves.

The steepening of whistler-mode waves has been recently studied for waves observed at the frequency of approximately 0.2 electron cyclotron frequency in the inner magnetosphere (Yoon et al. 2014), but it has been rarely studied in the past. The harmonic waves observed near the Moon can provide important clues for understanding the nonlinearity of whistler-mode waves in a plasma medium. Moreover, the lunar atmosphere and dust environment are investigated in the recent LADEE mission. We expect that the present study of the harmonic waves will expand our understanding of the intrinsic plasma behaviors around the Moon to the solar wind. The possibilities of the harmonics should be verified by comprehensive observations of multi-spacecraft in the future work. The quantitative effects on the waves and the steepening rate are left to be examined.

\section{Competing interests}

The authors declare that they have no competing interests.

\section{Authors' contributions}

YT analyzed the data and designed the research. YK and NT contributed to the interpretations. HT, FT, HS, HS, and MM acquired the data. All authors read and approved the final manuscript.

\footnotetext{
Acknowledgements

The authors wish to express their sincere thanks to all members of the Kaguya project team for processing and analyzing the data. This work was supported by a research fellowship of the Japan Society for the Promotion of Science for Young Scientists.
}

\section{Author details}

'Department of Geophysics, Graduate School of Science, Tohoku University, 6-3 Aramaki-aza-aoba, Aoba, Sendai, Miyagi 980-8578, Japan. ${ }^{2}$ Department of Earth and Planetary Sciences, Tokyo Institute of Technology, 2-12-1 Ookayama, Meguro, Tokyo 152-8551, Japan. ${ }^{3}$ Department of Earth and
Planetary Sciences, Faculty of Science, Kyushu University, 6-10-1 Hakozaki, Higashi-ku, Fukuoka 812-8581, Japan. ${ }^{4}$ Department of Earth and Environmental Sciences, Kumamoto University, 39-1 Kurokami, Kumamoto, Kumamoto 860-8555, Japan. ${ }^{5}$ Earthquake Research Institute, University of Tokyo, 1-1-1 Yayoi, Bunkyo-ku, Tokyo 113-0032, Japan.

Received: 18 June 2014 Accepted: 3 February 2015

Published online: 04 March 2015

\section{References}

Cattell C, Wygant JR, Goetz K, Kersten K, Kellogg PJ, von Rosenvinge T et al (2008) Discovery of very large amplitude whistler-mode waves in Earth's radiation belts. Geophys Res Lett 35:L01105, doi:10.1029/2007GL032009

Fairfield DH (1974) Whistler waves observed upstream from collisionless shocks. J Geophys Res 79:1368-78, doi:10.1029/JA079i010p01368

Hada T, Kennel CF, Terasawa T (1987) Excitation of compressional waves and the formation of shocklets in the Earth's foreshock. J Geophys Res 92:4423-35, doi:10.1029/JA092iA05p04423

Halekas JS, Brain DA, Mitchell DL, Lin RP (2006) Whistler waves observed near lunar crustal magnetic sources. Geophys Res Lett 33:L22104, doi:10.1029/ 2006GL027684

Katoh (2014) A simulation study of the propagation of whistler-mode chorus in the Earth's inner magnetosphere. Earth Planets Space 66:6, doi:10.1186/1880-5981-66-6

Kellogg PJ, Cattell CA, Goetz K, Monson SJ, Wilson LB (2011) Large amplitude whistlers in the magnetosphere observed with Wind-Waves. J Geophys Res 116:A09224, doi:10.1029/2010JA015919

Lembege B (2003) Full particle electromagnetic simulation of collisionless shocks. Space Plasma Simulation 615:54-78

Luo Q-Z, D'Angelo N, Merlino RL (1999) Experimental study of shock formation in a dusty plasma. Phys Plasmas 6:3455-8, doi:10.1063/1.873605

Orlowski DS, Russell CT (1995) Comparison of properties of upstream whistlers at different planets. Adv Space Res 16:137-41, doi:10.1016/0273-1177(95)00220-9

Russell CT (2007) Upstream whistler-mode waves at planetary bow shocks: a brief review. J Atmos Solar-Terrestrial Phys 69:1739-46, doi:10.1016/j.jastp.2006.11.004

Shimizu H, Takahashi F, Horii N, Matsuoka A, Matsushima M, Shibuya H, Tsunakawa H (2008) Ground calibration of the high-sensitivity SELENE lunar magnetometer LMAG. Earth Planets Space 60:353-63

Sonnerup BUÖ, Scheible M (1998) Minimum and maximum variance analysis ISSI. Sci Rep Ser 1:185-220

Stubbs TJ, Vondrak RR, Farrell WM (2006) A dynamic fountain model for lunar dust. Adv Space Res 37:59-66, doi:10.1016/j.asr.2005.04.048

Takahashi F, Shimizu H, Matsushima M, Shibuya H, Matsuoka A, Nakazawa S, lijima Y, Otake H, Tsunakawa H (2009) In-orbit calibration of the lunar magnetometer onboard SELENE (KAGUYA). Earth Planets Space 61:1269-74

Tsugawa Y, Katoh Y, Terada N, Ono T, Tsunakawa H, Takahashi F, Shibuya H, Shimizu H, Matsushima M (2014) Group-standing of whistler mode waves near the Moon. J Geophys Res 119:2634-48, doi:10.1002/2013JA019585

Tsugawa Y, Terada N, Katoh Y, Ono T, Tsunakawa H, Takahashi F, Shibuya H, Shimizu H, Matsushima M (2011) Statistical analysis of monochromatic whistler waves near the Moon detected by Kaguya. Ann Geophys 29:889-93, doi:10.5194/angeo-29-889-2011

Tsunakawa H, Shibuya H, Takahashi F, Shimizu H, Matsushima M, Matsuoka A, Nakazawa S, Otake H, lijima Y (2010) Lunar magnetic field observation and initial global mapping of lunar magnetic anomalies by MAP-LMAG onboard SELENE (Kaguya). Space Sci Rev 154:219-51, doi:10.1007/s11214-010-9652-0

Yoon P, Pandey V, Lee D (2014) Oblique nonlinear whistler wave. J Geophys Res 119:1851-62, doi:10.1002/2013JA018993.1 